\title{
Canciones y habilidades comunicativas orales, en estudiantes del primer grado de secundaria
}

Songs and oral communication skills, in first grade high school students

Canções e habilidades de comunicação oral em alunos do primeiro ano do ensino médio

\section{ARTÍCULO ORIGINAL}
Tabita Esther Carrillo Vargas
tabitacarrillo@upeu.edu.pe
https://orcid.org/0000-0001-6392-6810
Universidad Nacional de Educación Enrique
Guzmán y Valle, Lima - Perú

\author{
Maritza Mercedes Alejo Maraví \\ maritza_alma4@hotmail.com \\ https://orcid.org/0000-0003-0742-3682 \\ Guzmán y Valle, Lima - Perú
}
Universidad Nacional de Educación Enrique

Recibido 07 de Abril 2021 | Arbitrado y aceptado 19 de Junio 2021 | Publicado en 03 Setiembre 2021

\section{RESUMEN}

El presente estudio responde al objetivo demostrar que las canciones se relacionan con las habilidades comunicativas orales, en 50 estudiantes de inglés, del primer grado de secundaria de la Institución Educativa Adventista El Buen Pastor, Ñaña, en el 2014. La investigación es de tipo no experimental, sustantiva, estudio transversal y de diseño correlacional. Los instrumentos utilizados fueron dos: uno para medir el uso de las canciones y otro para las habilidades comunicativas orales. Según el análisis estadístico de Correlación de Pearson, el nivel de correlación es 0,678 puntos (moderada y positiva, superior a 0,286 ), y su valor de significancia es inferior a 0,05 ; es decir, la correlación es directa, moderada y significativa.

Palabras clave: canciones, habilidades comunicativas orales, habilidades perceptivas $\mathrm{y}$ habilidades productivas.

\section{ABSTRACT}

The present study responds to the objective of demonstrating that songs are related to oral communication skills, in 50 students of English, from the first grade of secondary school of the Adventist Educational Institution El Buen Pastor, Naña, in 2014. The research is of type not experimental, substantive, cross-sectional study and correlational design. The instruments used were two: one to measure the use of songs and another for oral communication skills. According to the statistical analysis of Pearson's Correlation, the level of correlation is 0.678 points (moderate and positive, greater than 0.286), and its significance value is less than 0.05 ; that is, the correlation is direct, moderate, and significant.

Keywords: songs, oral communication skills, perceptual skills and productive skills.

\section{RESUMO}

O presente estudo responde ao objetivo de demonstrar que as canções estão relacionadas às habilidades de comunicação oral, em 50 alunos ingleses, da primeira série do ensino médio da Instituição Educacional Adventista El Buen Pastor, Ñaña, em 2014. A pesquisa é do tipo estudo experimental, substantivo, transversal e desenho correlacional. Os instrumentos utilizados foram dois: um para mensurar o uso de músicas e outro para as habilidades de comunicação oral. De acordo com a análise estatística de Correlação de Pearson, o nível de correlação é de 0,678 pontos (moderado e positivo, maior que 0,286 ), e seu valor de significância é menor que 0,05 ; ou seja, a correlação é direta, moderada e significativa.

Palavras-chave: canções, habilidades de comunicação oral, habilidades perceptivas e habilidades produtivas. 


\section{INTRODUCCIÓN}

Las canciones y las habilidades comunicativas orales constituyen las inquietudes mayores del presente estudio; en el sentido, de estudiar la importancia de las canciones en inglés, para los estudiantes quienes necesitan cultivar y desarrollar sus habilidades comunicativas orales. Se ha trabajado las dos variables. La segunda: habilidades comunicativas orales, comprende las dimensiones: habilidades perceptivas orales y habilidades productivas orales.

En el espacio que determina el proceso enseñanza aprendizaje, existen tres conceptos muy importantes: intuición, experiencia y habilidades perceptivas, los cuales determinan capacidades y competencias en la parte de los estudiantes, quienes crece y desarrollan gracias a estas habilidades comunicativas (Romero 2012). Las habilidades y los conocimientos son elementos constitutivos de la alfabetización temprana, en los niños y los estudiantes; es decir, quienes tienen habilidades y conocimientos poseen ventajas académicas para sus aprendizajes, razón por la cual se subraya la relevancia de las habilidades y los conocimientos (Plana y Fumagalli 2013). Las personas y los estudiantes tienen un comportamiento visual muy importante para generar los aprendizajes, el cual es determinado por la percepción cognitiva; además, permite asumir y tomar decisiones; en el caso de los estudiantes, decisiones relacionadas con el aprendizaje (Vila-Maldonado, García López y Contreras Jordán, 2012). Existe, mejor dicho, se presenta un dilema en la educación con el propósito de lograr mejores aprendizajes, de desarrollar a los estudiantes en su proceso de enseñanza y aprendizaje; ¿se mejoran los aprendizajes mejorando el currículo?, ¿o mejorando las habilidades específicas de los estudiantes? Siempre el espacio educativo y sus ambientes so complejos (Oyarzún-Burgos 2014).

En este contexto académico y de aprendizaje, surge el objetivo de analizar la relación de las canciones con las habilidades comunicativas orales, en los estudiantes de inglés.

\section{Las canciones: su importancia}

Para Castellanos y Garzón (2013), la importancia de las canciones se manifiesta en su influjo sobre el desarrollo cognitivo y sobre los aspectos emocionales del niño. Las canciones son usadas en la condición de métodos terapéuticos (musicoterapia), sin perder de vista que la música influye sobre los dos hemisferios cerebrales, permite el desarrollo del córtex cerebral, genera muchas ventajas sobre los aprendizajes de los alumnos. Por su parte, Cordero (2014) afirma que las canciones y la música estimulan las inteligencias, contribuyen para las inteligencias múltiples, favoreciendo la didáctica de la enseñanza de 
las lengua extranjeras. Iacoboni, Jalo y Valotta (2014) afirman que las canciones cuentan historias; es decir, las canciones son recursos didácticos, mediante las canciones se exponen roles y usos reales de las lenguas extranjeras, entregando vocabularios, significados, estructuras gramaticales; también generan motivación, son denominadas metodologías prácticas para enseñar y aprender "elementos lingüísticoliterarios, socioculturales y musicales".

\section{Recursos, materiales, técnicas del uso de las canciones}

Según Leganés (2012), para la realización y el éxito del aprendizaje y la música a desarrollarse en las aulas, se requiere la creación de materiales con una perspectiva multidisciplinaria, contextualizando el valor de la música para la educación general y para el aprendizaje de lenguas extranjeras. Vaquero-González (2012) sostiene que la música y las canciones constituyen recursos de enseñanza y aprendizaje, una estrategia de aprendizaje durante los procesos de enseñanza de lengua extranjeras; estos recursos presentan ventajas y desventajas.

Para seleccionar las canciones, se debe tener en cuenta las condiciones académicas, psicológicas, culturales y sociales de los estudiantes. También la edad y las preferencias, los conocimientos previos, el nivel de estudios, los contenidos, la letra, la pronunciación, los acordes, los gustos musicales, aspectos motivacionales, los objetivos académicos, el ritmo, la contextualización (Castellanos y Garzón 2013). La música popular y las canciones en inglés se constituyen en herramientas de aprendizaje, con el propósito de trabajar la pronunciación y la gramática de la lengua extranjera: el inglés (Sánchez, 2014).

\section{Habilidades comunicativas}

Es necesario el acompañamiento del docente. Las habilidades comunicativas son: escuchar, hablar, leer y escribir. Todos los estudiantes, inclusive todas las personas tienen estas habilidades, las cuales son inherentes a las personas comunes y singulares. En este sentido, el profesor tiene que realizar prácticas para desarrollar y fortalecer estas habilidades, construir herramientas y estrategias para evaluar el desarrollo de las referidas habilidades. El docente debe recibir preparación, formación para lograr este rol, debe generar acompañamiento pedagógico (Vicente, 2012). La habilidades sociales y comunicativas son muy importantes, fortalecen a las personas, para se desenvolviendo en los diferentes espacios. Muchos estudiantes terminan sus carreras profesionales y no han desarrollado y fortalecido estas habilidades, quedando en la postergación y en el desaliento, incluso quedan marginados de los puestos de trabajo, no tienen acceso a una plaza, a un cargo, porque tienen problemas de comunicación (Abadanes Sánchez, 2014). 
Para Sanhueza et al (2012), en la comunidad, en las instituciones son necesarias las competencias comunicativas interculturales; las personas con estas competencias y habilidades marcan diferencia cultural, cognitiva, tienen potencial comunicativo, son suficientemente eficaces, en contextos sociales y culturales; exhiben su potencial reflexivo, han experimentado su desarrollo cognitivo, comportamental y altamente afectivo, han desarrollado su capacidad y habilidad de interacción e interrelación.

\section{METODOLOGÍA}

\section{Participantes}

En la investigación participaron 50 estudiantes del primer grado de secundaria de la Institución Educativa Adventista El Buen Pastor, Ñaña, el año 2014, quienes fueron elegidos y determinados por conveniencia.

\section{Tipo de investigación}

La investigación es no experimental, transversal, correlacional. No se han manipulado las variables. Se aplicó el instrumento haciendo un corte en el tiempo; además se determina la relación de las variables.

\section{Diseño de la investigación}

Esta investigación es de diseño correlacional, ya que se relaciona las variables estrategias de aprendizaje y rendimiento académico. Según Hernández Sampieri, Fernández Collado, and Baptista Lucio (2014), este tipo de estudio explica la relación o grado de asociación de dos o más conceptos, categorías, variables.

El diagrama representativo es el siguiente:

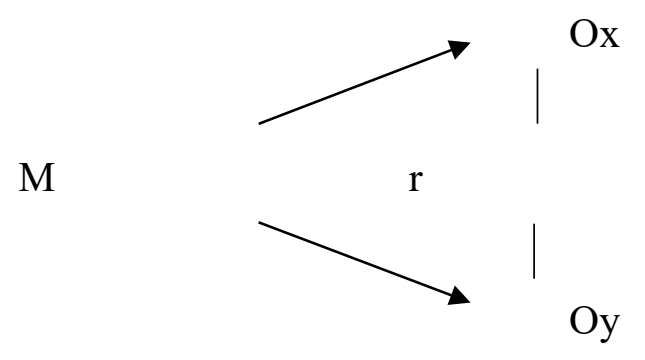


Donde:

M: Muestra de la investigación

0y: Observación de la variable I: Las canciones

0x: Observación de la variable II: Habilidades comunicativas orales

r : Relación entre las dos variables

\section{Instrumentos y técnicas de recolección}

Para medir y evaluar las variables se utilizaron: cuestionario, fichas bibliográficas o textuales, fichas de evaluación de expertos. Se usó la técnica: la encuesta, para recabar la información y los datos. También se hizo un análisis documental, el juicio de expertos. El instrumento presenta las alternativas tipo Likert: nunca (), casi nunca (2), algunas veces (3), a menudo (4), siempre (5), para el instrumento canciones; para el instrumento de habilidades comunicativas, las alternativas también son las mismas y con los mismos valores. Validación del instrumento

La validación de los instrumentos se realizó mediante la construcción de una prueba piloto y la opinión de expertos, siguiendo los lineamientos propuestos por Hernández Sampieri, Fernández Collado, and Baptista Lucio (2014). La validez de contenido fue calculada mediante el método de juicios de expertos. El informe de juicio de expertos se realizó con el apoyo de 4 docentes de la universidad con amplia experiencia en el campo de la investigación educacional. El promedio de la opinión de los expertos fue de 83,1 y 82,7 para los dos cuestionarios, respectivamente. Confiabilidad de los instrumentos

El criterio de confiabilidad del instrumento se determina, en la presente investigación, por el coeficiente Alfa de Cronbach, desarrollado por J. L. Cronbach, que requiere de una sola administración del instrumento de medición y produce valores que oscilan entre uno y cero. Es aplicable a escalas de varios valores posibles, por lo que puede ser utilizado para determinar la confiabilidad en escalas cuyos ítems tienen como respuesta más de dos alternativas. Su fórmula determina el grado de consistencia y precisión; la escala de valores que determina la confiabilidad está dada por los siguientes valores: 
Tabla 1

Criterio de confiabilidad

Criterio de confiabilidad

\section{Valores}

No es confiable

-1 a 0

Baja confiabilidad

0.01 a 0.49

Moderada confiabilidad

0.5 a 0.75

Fuerte confiabilidad

0.76 a 0.89

Alta confiabilidad $\quad 0.9$ a 1

\section{Análisis de fiabilidad}

Tabla 2

Criterio de confiabilidad Alpha de Cronbach

\begin{tabular}{lll}
\cline { 2 - 3 } Instrumento & Alfa de Cronbach & N de elementos \\
\hline Uso de las canciones &, 876 & 14 \\
Habilidades &, 810 & 12 \\
Comunicativas orales & & \\
\hline
\end{tabular}

El instrumento que mide el uso de canciones presenta fuerte confiabilidad con un valor del estadígrafo de $\mathbf{0 , 8 7 6}$ y con un instrumento que consta de 14 ítems. El instrumento que mide las habilidades comunicativas orales presenta fuerte confiabilidad con un valor del estadígrafo de $\mathbf{0 , 8 1 0}$ y con un instrumento que consta de 12 ítems. Como los instrumentos presentan adecuada confiabilidad en sus respectivas versiones, podemos concluir que los instrumentos son aplicables. 


\section{RESULTADOS}

\section{Prueba de hipótesis Hipótesis de investigación}

Las canciones se relacionan significativamente con las habilidades comunicativas orales en el idioma inglés en los estudiantes del primer grado de secundaria de la Institución Educativa Adventista El Buen Pastor, Ñaña, en el 2014”.

\section{Hipótesis nula}

Las canciones no se relacionan significativamente con las habilidades comunicativas orales en el idioma inglés en los estudiantes del primer grado de secundaria de la Institución Educativa Adventista El Buen Pastor, Ñaña, en el 2014. Hipótesis

\section{Estadística}

$H p: r>0.286$ Ho $: r £ 0.286 \mathrm{a}=$

0.05

Denota:

Hp: El índice de correlación entre las variables será superior a 0.286. Ho: El índice de correlación entre las variables será inferior o igual a 0.286. El valor de significancia estará asociado al valor $\alpha=0.05$

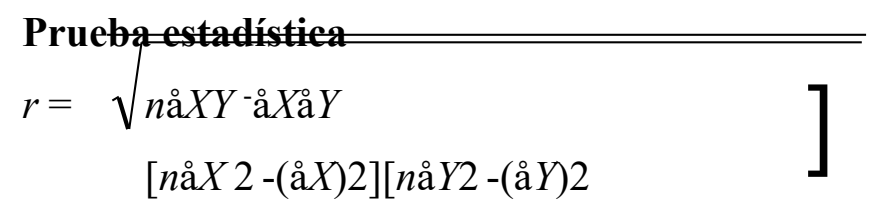

Determinación de la zona de rechazo de la hipótesis nula Nivel de confianza al 95\%

Valor de significancia: $\mathrm{a}=0.05$

Muestra: $50, \mathrm{gl}=50-2=48$

Zona de rechazo de la hipótesis nula: $\{r x y / r x y>0.286\}$

Tabla 3

Correlaciones de las canciones y habilidades comunicativas orales

Correlación de Pearson

Habilidades receptivas orales

La canciones

, $678^{* *}$

Sig. (bilateral)

, 000

$\mathrm{N}$ 
En la hipótesis general apreciamos que el nivel de correlación es de 0,678 puntos, moderada y positiva, superior a 0,286 , y su valor de significancia es inferior a 0,05 , es decir que la correlación es directa, moderada y significativa, al ser mayor al valor crítico de zona de rechazo de hipótesis nula podemos afirmar que existen razones suficientes para rechazar la hipótesis nula.

\section{Prueba de hipótesis específica $\mathrm{N}^{\circ} 1$ Hipótesis de investigación}

Las canciones se relacionan significativamente con las habilidades perceptivas orales en el idioma inglés en los estudiantes del primer año de secundaria de la Institución Educativa Adventista El Buen Pastor, Ñaña, en el 2014.

\section{Hipótesis nula}

Las canciones no se relacionan significativamente con las habilidades perceptivas orales en el idioma inglés en los estudiantes del primer año de secundaria de la Institución Educativa Adventista El Buen Pastor, Ñaña, en el 2014. Hipótesis estadística

$H p: r>0.286$

Ho : $r £ 0.286$

$\mathrm{a}=0.05$

Denota:

Hp: El índice de correlación entre las variables será superior a 0,286.

Ho: El índice de correlación entre las variables será inferior o igual a 0,286.

El valor de significancia estará asociado al valor $\alpha=0,05$

\section{Prueba estadística}

$n a ̊ X Y-a ̊ X a ̊ Y$

$r=$

[nåX $2-(a ̊ \gamma) 2][n a ̊ Y 2-(a ̊ Y) 2]$

Determinación de la zona de rechazo de la hipótesis nula Nivel de confianza al 95\%

Valor de significancia: $\mathrm{a}=0.05$

Muestra: $50, \mathrm{gl}=50-2=48$

Zona de rechazo de la hipótesis nula: $\left\{r_{x y} / r_{x y}>0.286\right\}$ 
Tabla 4

Correlaciones de las canciones y habilidades perceptivas orales

Correlación de Pearson

Habilidades receptivas orales

La canciones , $733^{* *}$

Sig. (bilateral)

, 000

$\mathrm{N}$

En la hipótesis específica $\mathrm{N}^{\circ} 1$ apreciamos que el nivel de correlación es de 0,733 puntos, fuerte y positiva, superior a 0,286 , y su valor de significancia es inferior a 0,05 , es decir que la correlación es directa, fuerte y significativa, al ser mayor al valor crítico de zona de rechazo de hipótesis nula, podemos afirmar que existen razones suficientes para rechazar la hipótesis nula.

\section{Prueba de hipótesis específica $\mathbf{N}^{\circ} 2$ Hipótesis de investigación}

Las canciones se relacionan significativamente con las habilidades productivas orales en el idioma inglés en los estudiantes del primer año de secundaria de la Institución Educativa Adventista El Buen Pastor, Ñaña, en el 2014.

\section{Hipótesis nula}

Las canciones no se relacionan significativamente con las habilidades productivas orales en el idioma inglés en los estudiantes del primer año de secundaria de la Institución Educativa Adventista El Buen Pastor, Ñaña, en el 2014.

\section{Hipótesis estadística}

$H p: r>0.286$

Ho : $r £ 0.286$

$\mathrm{a}=0.05$

Denota:

Hp: El índice de correlación entre las variables será superior a 0,286.

Ho: El índice de correlación entre las variables será inferior o igual a 0,286.

El valor de significancia estará asociado al valor $\alpha=0,05$ Prueba Estadística.

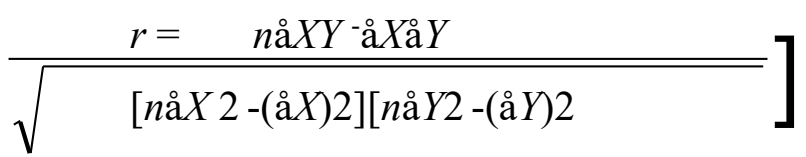


Determinación de la zona de rechazo de la hipótesis nula Nivel de confianza al 95\%

Valor de significancia: $\mathrm{a}=0.05$

Muestra: $50, \mathrm{gl}=50-2=48$

Zona de rechazo de la hipótesis nula: $\{r x y / r x y>0.286\}$

Tabla 5

Correlaciones de las canciones y habilidades productivas orales

Correlación de Pearson

Habilidades productivas orales

La canciones

, $494^{* *}$

Sig. (bilateral)

, 000

$\mathrm{N}$

En la hipótesis específica $\mathrm{N}^{\circ} 2$ apreciamos que el nivel de correlación es 0,494 puntos, moderada y positiva, superior a 0,286 , y su valor de significancia es inferior a 0,05 , es decir que la correlación es directa, moderada y significativa, al ser mayor al valor crítico de zona de rechazo de hipótesis nula, podemos afirmar que existen razones suficientes para rechazar la hipótesis nula.

\section{DISCUSIONES Y CONCLUSIONES}

Esta conclusión reafirma lo que Bonilla y otros (2007) encontraron en su investigación con respecto a las canciones en la adquisición de un idioma refiriéndose como una estrategia positiva que permite aprender nuevas palabras, la sonoridad, pronunciación y ritmo como que le permitirán a los estudiantes a recordar y predisponerlos hacia el desarrollo de sus habilidades comunicativas y, como Cuji y Rea (2011) dicen, sobre la necesidad que los docentes deben dar a conocer cuáles son las habilidades receptivas y productivas que se desarrolla dentro de los idiomas.

En la hipótesis específica $\mathrm{N}^{\circ} 1$, apreciamos que el nivel de correlación es de 0,733 puntos, fuerte y positiva, superior a 0,286 , y su valor de significancia es inferior a 0,05, es decir que la correlación es directa, fuerte y significativa. Al ser mayor al valor crítico de la zona de rechazo de la hipótesis nula, podemos afirmar que existen razones suficientes para rechazar la hipótesis nula, por lo que podemos inferir que: "Las canciones se relacionan significativamente con las habilidades perceptivas orales en el idioma inglés en los estudiantes del primer grado de secundaria de la Institución Educativa Adventista El Buen Pastor, Ñaña, en el 2014”. Valdez (2001) indica que las canciones son herramientas 
fundamentales en el aprendizaje debido a que forman parte de nuestra identidad, además de apoyarnos en el desarrollo y práctica de las habilidades auditivas, pronunciación y adquisición de vocabulario.

En la hipótesis específica $\mathrm{N}^{\circ} 2$, apreciamos que el nivel de correlación es de 0,494 puntos, moderada y positiva, superior a 0,286 , y su valor de significancia es inferior a 0,05 , es decir que la correlación es directa, moderada y significativa. Al ser mayor al valor crítico de la zona de rechazo de la hipótesis nula, podemos afirmar que existen razones suficientes para rechazar la hipótesis nula, por lo que podemos inferir que: "Las canciones se relacionan significativamente con las habilidades productivas orales en el idioma inglés en los estudiantes del primer grado de secundaria de la Institución Educativa Adventista El Buen Pastor, Ñaña, en el 2014”.

La investigación de Caro, Collana y Condori (2012) indica que las canciones influyen significativamente en el aprendizaje del idioma inglés, en las capacidades de expresión oral (habilidad productiva), comprensión de textos y producción de textos en los estudiantes, lo que reafirma nuestra hipótesis.

Existen razones suficientes para rechazar la hipótesis nula, por lo que podemos inferir que las canciones se relacionan significativamente con las habilidades comunicativas orales en el idioma inglés en los estudiantes del primer grado de secundaria de la Institución Educativa Adventista El Buen Pastor, Naña, en el 2014. Existen razones suficientes para rechazar la hipótesis nula; por eso se infiere que las canciones se relacionan significativamente con las habilidades perceptivas orales en el idioma inglés en los estudiantes del primer grado de secundaria de la Institución Educativa Adventista El Buen Pastor, Ñaña, en el 2014. Por otro lado, también existen sustentos y evidencias suficientes para rechazar la hipótesis nula; en consecuencia, se puede inferir que las canciones se relacionan significativamente con las habilidades productivas orales en el idioma inglés en los estudiantes del primer grado de secundaria de la Institución Educativa Adventista El Buen Pastor, Ñaña, en el 2014.

En la hipótesis general, apreciamos que el nivel de correlación es de 0,678 puntos, moderada y positiva, superior a 0,286 , y su valor de significancia es inferior a 0,05 , es decir que la correlación es directa, moderada y significativa, al ser mayor al valor crítico de zona de rechazo de hipótesis nula, podemos afirmar que existen razones suficientes para rechazar la hipótesis nula. Por lo tanto, existen razones suficientes para rechazar la hipótesis nula, por lo que podemos inferir que "Las canciones se relacionan significativamente con las habilidades comunicativas orales en el idioma inglés en los 
estudiantes del primer grado de secundaria de la Institución Educativa Adventista El Buen

Pastor, Ñaña, en el 2014”.

\section{REFERENCIAS}

Abadanes Sánchez, Marta. 2014. “Utilizar Las Tecnologías Para Potenciar Las

Habilidades Sociales Y Comunicativas." Historia Y Comunicación Social

19(Especial):679-88. Retrieved

(https://abacus.universidadeuropea.es/bitstream/handle/11268/8072/abanades_201

4.pdf? sequence $=2 \&$ isAllowed $=y$ ).

Castellanos, Luisa and Yina Garzón. 2013. "El Uso de Canciones En Inglés Para

Favorecer El Desarrollo de Habilidades de Comprensión.” [Tesis de título profesional, Universidad Libre de Colombia]. Retrieved

(https://repository.unilibre.edu.co/bitstream/handle/10901/7898/CastellanosEspitia

LuisaFernanada2013.pdf?sequence=1).

Cordero, Dayane. 2014. "Las Canciones Mueven Tus Inteligencias.” Foro de

Profesores de E/LE (10):43-53. Retrieved

(https://dialnet.unirioja.es/servlet/articulo?codigo=4859798).

Hernández Sampieri, Roberto, Carlos Fernández Collado, and Maria del Pilar Baptista

Lucio. 2014. Metodología de La Investigación. Sexta edic. México, D.F:

McGrawHill Interamericana Editores, S.A. de C.V.

Iacoboni, Gabriela, Marcela Jalo, and María Valotta. 2014. “Canciones Que Cuentan

Historias." Puertas Abiertas (10):1-11. Retrieved

(http://www.memoria.fahce.unlp.edu.ar/art_revistas/pr.6374/pr.6374.pdf).

Leganés, Esther. 2012. "La Música En El Aula de Inglés: Una Propuesta Práctica."

Encuentro. Revista de Investigación E Innovación En La Clase de Idiomas

(21):115-25. Retrieved (https://core.ac.uk/download/pdf/58909657.pdf).

Oyarzún-Burgos, Carlos. 2014. "El Dilema de La Educación Diferencial Hoy: ¿el

Currículum O Las Habilidades Específicas?” Revista Temas de Educación

19(2):69-79. Retrieved

(https://revistas.userena.cl/index.php/teduacion/article/view/448).

Plana, María and Julieta Fumagalli. 2013. "Habilidades Y Conocimientos Constitutivos de La Alfabetización Temprana: Semejanzas Y Diferencias Según El Entorno Social Y Las Oportunidades Educativas." Interdisciplinaria 30(1):5-24. Retrieved 
(https://ri.conicet.gov.ar/bitstream/handle/11336/28701/CONICET_Digital_Nro.d2 681c80-4a4c-43ce-b899-4286d910faf0_A.pdf?sequence=2).

Romero, Ignacio. 2012. "El Portero de Balonmano Y Las Habilidades Perceptivas: Propuesta de Entrenamiento En Niños.” E-Balonmano.com: Revista de Ciencias Del Deporte 8(3):209-22. Retrieved (http://dehesa.unex.es/bitstream/handle/10662/7005/18857019_8_3_209.pdf?seque nce $=1 \&$ isAllowed $=\mathrm{y})$.

Sánchez, Jorge. 2014. "Enseñando Inglés Con Canciones.” Revista Ciencias Humanas 11:46-64. Retrieved

(https://revistas.usb.edu.co/index.php/CienciasHumanas/article/view/1805/1563).

Sanhueza, Susan, Fraño Paukner, Víctor San Martín, and Miguel Friz. 2012.

"Dimensiones de La Competencia Comunicativa Intercultural (CCI) Y Sus

Implicaciones Para La Práctica Educativa Dimensions of Intercultural

Communicative Competence (ICC) and Its Implications for Educational Practice."

Revista Folios (36):131-51. Retrieved

(https://www.redalyc.org/pdf/3459/345932039008.pdf).

Vaquero-González, Miriam. 2012. “La Canción Como Recurso Didáctico En El Aula de Lengua Extranjera." [Tesis de grado, Universidad de Valladolid]. Retrieved (https://uvadoc.uva.es/bitstream/handle/10324/2099/TFG-L+130.pdf?sequence=1 ) .

Vicente, Macario. 2012. “Impacto Del Acompañamiento Pedagógico En Las Prácticas Del Docente de Primer Grado Primario Bilingüe En El Desarrollo de Las

Habilidades Comunicativas En Idioma Materno K'iche' En Municipios de Quiché." [Tesis de título profesional, Universidad Rafael Landívar]. Retrieved (file:///E:/Habilidades comunicativas/Vicente-Macario.pdf).

Vila-Maldonado, S., L. M. García López, and O. R. Contreras Jordán. 2012. “La Investigación Del Comportamiento Visual, Desde El Enfoque PerceptivoCognitivo Y La Toma de Decisiones En El Deporte.” Journal of Sport and Health Research 4(2):13756. Retrieved (http://journalshr.com/papers/Vol 4_N 2/V04_2_4.pdf). 